\title{
Recurrent Herpes simplex virus infection after AstraZeneca vaccination for covid 19: A case report
}

\author{
Mohammadreza ardalan ${ }^{1}$, Hamidreza Moslemi ${ }^{2}$, Shervin Shafiei ${ }^{2}$, Reza Tabrizi ${ }^{3}$, and \\ mohammadreza moslemi ${ }^{4}$ \\ ${ }^{1}$ Tabriz University of Medical Sciences \\ ${ }^{2}$ Shahid Beheshti University of Medical Sciences School of Dentistry \\ ${ }^{3}$ Shahid Beheshti University of Medical Sciences \\ ${ }^{4}$ Tabriz University of Medical Sciences Faculty of Medicine
}

July 13, 2021

\begin{abstract}
Vaccination against covid 19 have been launched by many countries. Since then, viral infection after vaccination have been reported. Herpes virus reactivation has been reported following vaccinations. This study reports a case of recurrent Herpes Simplex Virus (HSV) after chadox1 ncov-19 (azd1222) vaccine in a 28 years old man
\end{abstract}

\section{Introduction}

Since covid19 pandemic, safe and effective vaccination against it became so important. Up to now, more than 50 vaccines against severe acute respiratory syndrome coronavirus 2 (SARS-CoV-2) have been developed in clinical trials. Chadox1 ncov-19 (AZD1222) is one of them which has been used in national vaccination programs against the disease in many countries ${ }^{1}$. Viral infection after covid 19 vaccination have been reported. Furer et al, has reported six cases of herpes zoster following BNT162b2 mRNA Covid-19 vaccination 2 .

Herpes virus reactivation has been reported following trivalent influenza, hepatitis A, and rabies vaccines, suggesting vaccine-modulated immunomodulation ${ }^{3}$. To our knowledge there were no case reports of herpes simplex infections or herpes like skin lesions in the chadox1 ncov-19 (azd1222) vaccine clinical trials ${ }^{4}$, but some cases have been reported in the UK spontaneous reports received between April $1^{\text {st }}$ to April $14^{\text {th }} 2021$ for COVID-19 vaccine Oxford University/AstraZeneca.

In this study we are going to report a Herpes simplex virus (HSV) reactivation following chadox1 ncov-19 (AZD1222) vaccination.

\section{Case Presentation}

A 28 years old man, with no systemic disease, presented with recurrent HSV lesion on the right upper eye lid. He received first dose of chadox1 ncov-19 (AZD1222) vaccine on 20th March 2021 during Iran national vaccination program. Besides the common post-vaccination signs and symptoms, two days later, burning pain and blistery skin rashes developed on the right upper eye lid (Figure 1). Ocular examination was done by slit-lamp which showed normal findings. Upper eye lid edema occurred three days later (Figure 2). Along with these findings, patient remembered an infection with HSV following a trauma to the right eye with a hand watch in his childhood. According to the patient, he has experienced periods of cold sore (herpes simplex labialis) after that. Regarding clinical signs and symptoms and the history of the patient, treatment was started based on the diagnosis of recurrent HSV lesion of the upper eye lid. Topical 
therapy was instructed to the patient including Eye Drop Sulfacetatamide 10\% to treat probable bacterial conjectivitis and blepharitis because of severe periorbital edema and Eye Drop Ketotifen and Vitamin A ointment to reduce itching and irritation. 72 hours later, patient reported blurred vision of the right eye, which ocular examinations were normal again. Seven days after the onset of the lesion, signs and symptoms got disappeared. Written informed consent was obtained from the patient for to the publication of this report and clinical images.

\section{Discussion}

We reported a 28 years old patient who developed Herpes simplex virus as vesicular ulcerative periorbital lesion 48 hours after the first dose chadox1 ncov-19 (Azd1222) vaccination against covid19.

Since ongoing covid19 pandemic, herpes simplex or herpes zoster like lesions have been reported during covid19 infection ${ }^{5,6}$. This might be explained through function of Natural Killer Group 2D (NKG2D) ligands which is also known as "stress-induced ligands", found on healthy cells, such as neuronal cells, in order to avoid auto-reactivity of natural killer T cells against normal tissues. HSV-1 and HSV-2 cause downregulation of NKG2D ligands on neural cell membranes and terminals after infection of these cells, so the infected cells could not be recognized with natural killer cells and the HSV infection enters its latent phase $^{7}$. These ligands, however, are upregulated on the cell surface following various stresses including oncogene activation, hypoxia or viral infections ${ }^{8,9}$ and the last two conditions are present in covid19 which can explain the coincidence of covid 19 infection and HSV reactivation.

Also, ChAdOx1 nCoV-19 (AZD1222) is a replication-deficient simian adenoviral vector that expresses the full-length SARS-CoV-2 spike protein. Ewer et al. ${ }^{10}$ showed that this vaccine can initiate cytokine release and immune response cascades in a similar way to viral infections and SARS-CoV-2. This mechanism can lead to upregulation of NKG2D ligands and reactivation of the HSV from its latent phase and developing of the clinical signs and symptoms of herpes labialis after vaccination.

The diagnosis of mucocutaneous involvement of HSV and its differentiation from Herpes Zoster infection was based on clinical signs, which was one of the limitations of this report. Serologic and molecular virus detection tests should have been done for definite diagnosis.

This study revealed a possible relation between chadox1 ncov-19 (azd1222) vaccination and HSV reactivation which also cannot be proved based on this report, alone. Awareness should be raised regarding the potential link between COVID-19 infection or vaccination and HSV reactivation. Further similar reports and safety monitoring studies on chadox1 ncov-19 (azd1222) and other COVID-19 vaccination side effects is required for more absolute conclusion.

\section{Acknowledgment}

None

\section{Funding}

This research did not receive any specific grant from funding agencies in the public, commercial, or not-forprofit sectors.

\section{Conflicts of interest}

None

\section{Ethics}

This study was performed according to the principles outlined by the World Medical Association's Declaration of Helsinki on experimentation involving human subjects, as revised in 2000 and has been approved by the ethics committee of the Tabriz University of Medical Sciences.

\section{Author Contributions:}


Mohammadreza Ardalan: The conception and design of the study

Hamidreza Moslemi: Drafting the article or revising it critically for important intellectual content

Shervin Shafiei: Drafting the article or revising it critically for important intellectual content

Reza Tabrizi: Final approval of the version to be submitted

Mohammadreza Moselmi: The conception and design of the study, Final approval of the version to be submitted

\section{References}

1. Voysey M, Clemens SAC, Madhi SA, Weckx LY, Folegatti PM, Aley PK, et al. 2021 Safety and efficacy of the ChAdOx1 nCoV-19 vaccine (AZD1222) against SARS-CoV-2: an interim analysis of four randomised controlled trials in Brazil, South Africa, and the UK. Lancet 397:99-111.

2. Furer V, Zisman D, Kibari A, Rimar D, Paran Y, Elkayam O. 2021 Herpes zoster following BNT162b2 mRNA Covid-19 vaccination in patients with autoimmune inflammatory rheumatic diseases: a case series. Rheumatology (Oxford).

3. Walter R, Hartmann K, Fleisch F, Reinhart WH, Kuhn M. 1999 Reactivation of herpesvirus infections after vaccinations? The Lancet 353:810.

4. Kim SH, Wi YM. 2021 Adverse Events in Healthcare Workers after the First Dose of ChAdOx1 nCoV-19 or BNT162b2 mRNA COVID-19 Vaccination: a Single Center Experience. J Korean Med Sci 36:e107.

5. Fernandez-Nieto D, Ortega-Quijano D, Suarez-Valle A, Burgos-Blasco P, Jimenez-Cauhe J, FernandezGuarino M. 2020 Comment on: "To consider varicella-like exanthem associated with COVID-19, virus varicella zoster and virus herpes simplex must be ruled out. Characterization of herpetic lesions in hospitalized COVID-19 patients". J Am Acad Dermatol 83:e257-e9.

6. Le Balc'h P, Pinceaux K, Pronier C, Seguin P, Tadié J-M, Reizine F. 2020 Herpes simplex virus and cytomegalovirus reactivations among severe COVID-19 patients. Crit Care 24:1-3.

7. Schepis D, D'amato M, Studahl M, Bergström T, Kärre K, Berg L. 2009 Herpes simplex virus infection downmodulates NKG2D ligand expression. Scand J Immunol 69:429-36.

8. Nausch N, Cerwenka A. 2008 NKG2D ligands in tumor immunity. Oncogene 27:5944-58.

9. González S, López-Soto A, Suarez-Alvarez B, López-Vázquez A, López-Larrea C. 2008 NKG2D ligands: key targets of the immune response. Trends Immunol 29:397-403.

10. Ewer KJ, Barrett JR. 2021 T cell and antibody responses induced by a single dose of ChAdOx1 nCoV-19 (AZD1222) vaccine in a phase 1/2 clinical trial. Nat Med 27:270-8.

\section{Figure legends}

Fig 1. Blistery skin rashes developed on the right upper eye lid two days after vaccination with Chadox1 ncov-19 (AZD1222)

Fig 2. Edema of the upper eye lid five days after vaccination with Chadox1 ncov-19 (AZD1222) 

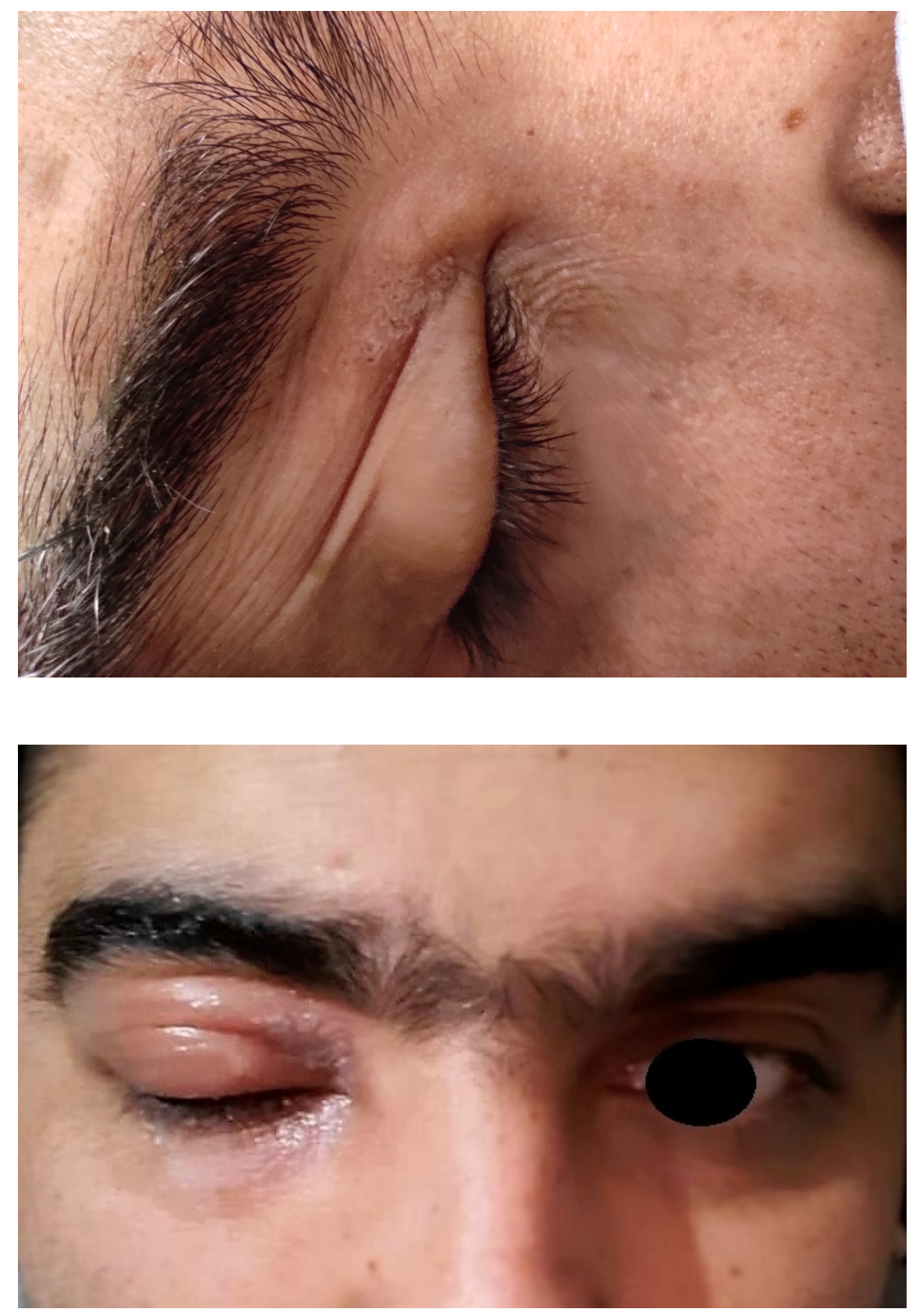